\title{
Sunitinib achieved fast and sustained control of VIPoma symptoms
}

\section{Louis de Mestier, Thomas Walter ${ }^{1}$, Hedia Brixi, Catherine Lombard-Bohas ${ }^{1}$ and Guillaume Cadiot}

Department of Hepato-Gastroenterology and Digestive Oncology, Hôpital Robert Debré, Boulevard du Général Koenig, 51100 Reims Cedex, France and ${ }^{1}$ Department of Gastroenterology and Digestive Oncology, Hospices Civils de Lyon, Hôpital Edouard Herriot, Lyon, France

\author{
Correspondence \\ should be addressed \\ to $\mathrm{G}$ Cadiot \\ Email \\ gcadiot@chu-reims.fr
}

\begin{abstract}
VIPomas are rare-functioning neuroendocrine tumors (NETs). Overproduction of vasointestinal peptide (VIP) leads to the VernerMorrison syndrome, whose management is challenging when refractory to somatostatin analogs. Two patients with progressive metastatic pancreatic NETs and refractory VIPoma symptoms were treated with sunitinib. This led to fast and sustained total relief of VIPoma symptoms, enabling earlier discharge from hospital and improvement in their quality of life. In both cases, sunitinib discontinuation led to the quick recurrence of watery diarrhea, which resolved within a few days after reintroducing sunitinib. The anti-secretory effect of sunitinib on VIPoma syndrome was probably not related to any anti-tumor effect. These observations agree with the rare reported cases of anti-secretory effects with targeted therapies. The sunitinibdriven inhibition of multiple-tyrosine kinase receptors might act on secretory pathways and describe sunitinib's ability to improve VIPoma symptoms. Sunitinib could be a therapeutic option to control refractory VIPoma symptoms in patients with NETs.
\end{abstract}

\section{Introduction}

VIPomas are rare-functioning neuroendocrine tumors (NETs) usually located in the pancreas. Vasointestinal peptide (VIP) overproduction leads to the VernerMorrison syndrome of high-volume watery diarrhea persisting upon fasting, often responsible for complications (hypokalemia, acidosis, dehydration) $(1,2)$. The management of VIPoma symptoms is challenging. When surgical resection of all the lesions is not feasible, symptom control is mainly based on somatostatin analogs (SSA) (1).

Sunitinib, a tyrosine-kinase inhibitor, effectively controlled the growth of pancreatic NETs, even though it did not significantly prolong progression-free survival of patients with functioning NETs in the pivotal trial (3). Specific data on sunitinib administration in VIPoma patients are scarce. We recently treated two patients with progressive metastatic pancreatic NETs and uncontrolled VIPoma symptoms, in whom sunitinib obtained complete, fast, and sustained anti-secretory effects.

\section{Clinical observation 1}

Patient 1 (25-year-old woman with negative-MEN1 screening) had an initially non-functioning metastatic grade 2 (Ki67 =19\%), moderately differentiated pancreatic NET, which had initially been treated with systemic chemotherapy, surgery of the primary tumor, and thermoablation of hepatic metastases. VIPoma symptoms developed 3 years later in a context of increased disease aggressiveness $(\mathrm{Ki} 67=40 \%)$ and consisted of 8-15 daily watery bowel movements associated with vomiting, diffuse abdominal pain, 5-kg weight loss, and hypokalemia (1.5 mmol/l). Despite high doses of i.v. SSA (octreotide $2000 \mu \mathrm{g} /$ day), liver chemoembolization and even partial surgical liver debulking, uncontrolled functional symptoms persisted. Because the disease was progressive, sunitinib (37.5 mg/day) was given which resolved the VIPoma syndrome within 4 days of treatment onset, with one daily bowel movement and normal plasma potassium levels.

Published by Bioscientifica Ltd. 


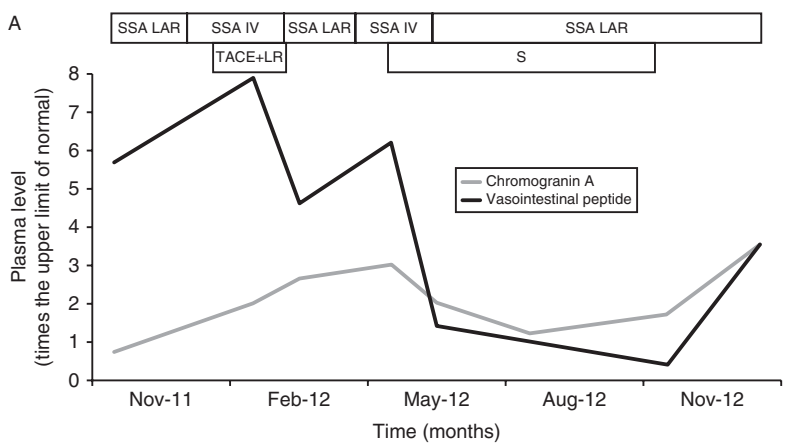

B
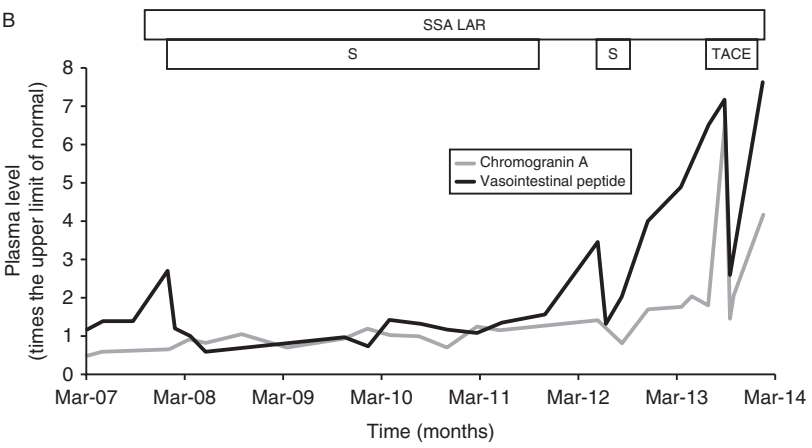

\section{Figure 1}

Evolution of chromogranin $A$ and vasointestinal peptide in patients $1(A)$ and 2 (B) who received sunitinib for pancreatic NETs and VIPoma symptoms. LR, liver resection; $S$, sunitinib; SSA, somatostatin analog (IV, i.v.; LAR, long-acting release); TACE, transarterial chemoembolization.

Within 1 month, the patient gained weight and the plasma VIP level declined dramatically with normalization, enabling the switch to long-acting release (LAR) lanreotide (60 mg/28 days) (Fig. 1A). After 7 months, because NETs were stable on computed-tomography images, sunitinib was discontinued because of vomiting, leading to the recurrence of watery diarrhea. Sunitinib ( $25 \mathrm{mg} /$ day) was reintroduced, which resolved diarrhea within 2 days. The patient died 3 months later due to denutrition and sepsis.

\section{Clinical observation 2}

Patient 2 (47-year-old man with negative-MEN1 screening) had a metastatic liver recurrence 5 years after surgical resection of a well-differentiated grade $2(\mathrm{Ki} 67=4 \%)$ pancreatic NETs. He complained of severe VIPoma symptoms of seven daily bowel movements, despite SSA (lanreotide LAR $120 \mathrm{mg} / 28$ days). Sunitinib (37.5 mg/day) achieved a rapid (3 days) and sustained clinical response, with one daily bowel movement without potassium supplementation. Biological response and disease stability were maintained with good tolerance (Fig. 1B). Sunitinib was stopped after 4 years (physician's decision), and 1 week later high-volume diarrhea and hypokalemia $(2.4 \mathrm{mmol} / \mathrm{l})$ reoccurred, with dehydration and requiring hospitalization. Sunitinib (37.5 mg/day) was restarted, obtaining functional symptom relief in 1 day. VIPoma symptoms recurred 3 months later and the patient underwent chemoembolization with moderate clinical efficacy.

\section{Discussion}

We observed rapid, complete and sustained resolutions of VIPoma-related watery diarrhea after the introduction of sunitinib for two patients. These responses enabled SSA-dose lowering, hypokalemia and dehydration reversals, hospital discharge, weight regain, and improved quality of life. The anti-secretory effect of sunitinib on VIPoma syndrome was probably not related to any anti-tumor effect, because: i) symptom relief occurred within a few days; ii) sunitinib interruption led to rapid recurrence of watery diarrhea, which resolved after its reintroduction, even at reduced dose; and iii) sunitinib induced dramatic biological responses as assessed by decreased plasma VIP and chromogranin A levels.

Although the pivotal clinical trial on sunitinib for pancreatic NETs included functioning tumors (3), the two patients with a VIPoma were randomized in the placebo arm and no data are currently available concerning the outcomes of VIPoma patients treated by sunitinib, particularly regarding functional control. Nevertheless, the current observations agree with the rare reported cases of anti-secretory effects with targeted therapies $(4,5,6,7)$.

Sunitinib's ability to improve NETs-related hormonal syndromes, in general, and VIPomas in particular, has never been specifically explored before, and is not yet understood. An intriguing observation for the two present cases was the swift control of the secretory syndrome by sunitinib with striking concordance between drug administration and symptoms. The time course is poorly consistent with a sole anti-angiogenic effect, but opens the possibility of a direct effect on the release of VIP from secretory granules in view of the simultaneous decrease in plasma VIP and chromogranin A concentrations. Indeed, VIP is stored in the secretory granules in pancreatic VIPomas (8). Of potential interest, in spite of rarity, is the fact that sunitinib was reported to rapidly inhibit the inappropriate hormone secretion by diverse types of VIP-producing NETs, including pancreatic VIPomas ((7), and the present cases), paraganglioma (7), and pheochromocytomas (9), suggesting an impact of the drug on hormone release. Little is known about the 
regulation of VIP secretion by tumor cells, beside the fact that both cyclic adenosine monophosphate and intracellular calcium appear to be stimulatory (10).

So far, the anti-tumoral effect of sunitinib has primarily been attributed to the inhibition of angiogenesis, through the blockade of several receptor tyrosine kinases. However, direct effects on intracellular pathways have also been described in some tumor types, among which NETs. Such a multi-targeted action has been reported in pheochromocytoma PC12 cells, including a reduction in inositol 1,4,5trisphosphate, which normally regulates hormone secretion through the increase in intracellular calcium rate (11). Besides, one well-described anti-secretory mechanism - among others - of SSAs is the decrease in intracellular calcium rate through the inhibition of adenyl cyclase and cyclic adenosine monophosphate, and through the activation of calcium channels (12). Hence, the interaction of sunitinib and SSAs could be synergistic for inhibiting hormonal hypersecretions in NETs in general and VIPomas in particular.

In spite of their rarity, VIP-secreting NETs may deserve special attention with respect to the potential benefit of sunitinib, alone or combined with SSAs, in order to assess whether this drug could become a first-order therapy for the Verner-Morrison syndrome, and to investigate the mechanism(s) at play in the blockade of VIP release.

For patient 1 , consent could not be obtained because the patient is deceased. For patient 2 , informed consent was obtained from the patient for publication of the case report.

\section{Declaration of interest}

L de Mestier: Pfizer (board). T Walter: Pfizer, Novartis, Ipsen, Celgen (board). H Brixi: Novartis, Ipsen (board). C Lombard-Bohas: Novartis, Ipsen, Pfizer, Keocyt (board). G Cadiot: Pfizer, Ipsen, Novartis (board).

\section{Funding}

This research did not receive any specific grant from any funding agency in the public, commercial or not-for-profit sector.

\section{Acknowledgments}

The authors thank Ms Janet Jacobson for editorial assistance in the preparation of the manuscript and Prof. Jean-Alain Chayvialle for his helpful comments.

\section{References}

1 Jensen RT, Cadiot G, Brandi ML, de Herder WW, Kaltsas G, Komminoth P, Scoazec J-Y, Salazar R, Sauvanet A \& Kianmanesh R. ENETS consensus guidelines for the management of patients with digestive neuroendocrine neoplasms: functional pancreatic endocrine tumor syndromes. Neuroendocrinology 201295 98-119. (doi:10.1159/ 000335591)

2 Smith SL, Branton SA, Avino AJ, Martin JK, Klinger PJ, Thompson GB, Grant CS \& van Heerden JA. Vasoactive intestinal polypeptide secreting islet cell tumors: a 15-year experience and review of the literature. Surgery 1998124 1050-1055. (doi:10.1067/msy.1998.92005)

3 Raymond E, Dahan L, Raoul J-L, Bang Y-J, Borbath I, Lombard-Bohas C, Valle J, Metrakos P, Smith D, Vinik A et al. Sunitinib malate for the treatment of pancreatic neuroendocrine tumors. New England Journal of Medicine 2011364 501-513. (doi:10.1056/NEJMoa1003825)

4 Kulke MH, Bergsland EK \& Yao JC. Glycemic control in patients with insulinoma treated with everolimus. New England Journal of Medicine 2009360 195-197. (doi:10.1056/NEJMc0806740)

5 Bourcier ME, Sherrod A, DiGuardo M \& Vinik AI. Successful control of intractable hypoglycemia using rapamycin in an 86-year-old man with a pancreatic insulin-secreting islet cell tumor and metastases. Journal of Clinical Endocrinology and Metabolism 200994 3157-3162. (doi:10.1210/ jc.2009-0788)

6 Bernard V, Lombard-Bohas C, Taquet M-C, Caroli-Bosc F-X, Ruszniewski P, Niccoli P, Guimbaud R, Chougnet CN, Goichot B, Rohmer $\mathrm{V}$ et al. Efficacy of everolimus in patients with metastatic insulinoma and refractory hypoglycemia. European Journal of Endocrinology 2013168 665-674. (doi:10.1530/EJE-12-1101)

7 Bourcier ME \& Vinik AI. Sunitinib for the treatment of metastatic paraganglioma and vasoactive intestinal polypeptide-producing tumor (VIPoma). Pancreas 201342 348-352. (doi:10.1097/MPA. Ob013e31825c53fa)

8 Solcia E, Capella C, Riva C, Rindi G \& Polak JM. The morphology and neuroendocrine profile of pancreatic epithelial VIPomas and extrapancreatic, VIP-producing, neurogenic tumors. Annals of the New York Academy of Sciences $1988 \mathbf{5 2 7}$ 508-517. (doi:10.1111/j.1749-6632. 1988.tb27004.x)

9 Lebowitz-Amit R, Mete O, Asa SL, Ezzat S \& Joshua AM. Malignant pheochromocytoma secreting vasoactive intestinal peptide and response to sunitinib: a case report and literature review. Endocrine Practice 201420 145-150. (doi:10.4158/EP14093.CR)

10 Harty RF, Jain DK \& McGuigan JE. Mechanisms of vasoactive intestinal peptide release in short-term culture of vasoactive intestinal peptideproducing tumor. Gastroenterology 198894 513-518. (doi:10.5555/ uri:pii:0016508588904465)

11 Aita Y, Ishii KA, Saito Y, Ikeda T, Kawakami Y, Shimano H, Hara H \& Takekoshi K. Sunitinib inhibits catecholamine synthesis and secretion in pheochromocytoma tumor cells by blocking VEGF receptor 2 via PLC- $\gamma$-related pathways. American Journal of Physiology. Endocrinology and Metabolism 2012303 1006-1014. (doi:10.1152/ajpendo.00156. 2012)

12 Modlin IM, Pavel M, Kidd M \& Gustafsson BI. Review article: somatostatin analogues in the treatment of gastroenteropancreatic neuroendocrine (carcinoid) tumours. Alimentary Pharmacology \& Therapeutics 201031 169-188. (doi:10.1111/j.1365-2036.2009.04174.x)

Received 10 August 2014

Revised version received 18 September 2014

Accepted 10 October 2014 